International Journal of Biomedicine | June 2021 - Volume 11, Issue Suppl_1: Abstracts from the Third Russian International Conference "Cryo-electron microscopy 2021: achievements and prospects"

POSTER ABSTRACT PRESENTATIONS

SESSION TITLE: STRUCTURE AND FUNCTIONS OF THE TRANSCRIPTION AND TRANSLATION APPARATUS OF THE CELL

DOI: 10.21103/IJBM.11.Suppl_1.P20

\title{
Abstract P-20: Sub $3 \AA ̊$ Resolution Cryo-EM Structure of Eukaryotic Small (40S) Ribosomal Subunit
}

$\underline{\text { Timur N. Baymukhametov }}^{1}$, Olesya V. Kravchenko ${ }^{2}$, Zhanna A. Afonina ${ }^{2}$, Konstantin S. Vassilenko ${ }^{2}$

\author{
${ }^{1}$ National Research Center "Kurchatov Institute," Moscow, Russia \\ ${ }^{2}$ Institute of Protein Research, Russian Academy of Sciences, Pushchino, Russia
}

Background: The ribosome is a molecular machine that translates mRNAs into proteins. In eukaryotes, ribosome consists of small (40S) and large (60S) subunits. Translation in eukaryotes is a complicated molecular process that involves the formation of various molecular complexes consisting of ribosomal subunits and protein factors. Cryo-EM approaches such as single particle analysis are widely used for structural analysis of components and intermediates of the translation machinery. However, the process of translation in plants is still poorly characterized at a structural level. Here, we present the structure of Triticum aestium small ribosomal subunit obtained at sub $3 \AA$ resolution that can be used for further structural studies of the translation process in plants.

Methods: The structures of the $40 \mathrm{~S}$ subunits purified from wheat germ extract were obtained using high-resolution single particle cryo-EM. For cryo-EM sample preparation were used Quantifoil R 1.2/1.3 grids coated with an additional $2 \mathrm{~nm}$ amorphous carbon film were glow-discharged for 30 seconds at $15 \mathrm{~mA}$ using PELCO easiGlow (Ted Pella). $3 \mu \mathrm{L}$ of the sample were applied onto the grids, blotted for $3 \mathrm{sec}$ at $10^{\circ} \mathrm{C}$ and $100 \%$ humidity, and plunge-frozen in liquid ethane using Vitrobot Mark IV (Thermo Fisher).

Cryo-EM data were collected using a $\mathrm{C}_{\mathrm{s}}$-corrected Titan Krios (Thermo Fisher) transmission electron microscope, equipped with a Falcon II direct electron detector. Data were acquired with defocus range of -0.6 to -2.0 at a nominal magnification of $75,000 x$, giving a calibrated pixel size of $0.86 \AA$ pixel. The micrographs were recorded as movie stacks. The exposure time for each stack 
was $1.6 \mathrm{~s}$, corresponding to a total electron dose of $\sim 84 \mathrm{e}^{-} / \AA^{2}$ fractionated into 32 frames $\left(\sim 2.6 \mathrm{e}^{-} / \AA^{2}\right.$ per single frame $)$. A total of 5521 movie stacks was collected. Raw cryo-EM data preprocessing was performed with Warp software (Tegunov et al., 2019). All further data processing steps were performed using the cryoSPARC v3.2.0 software (Punjani et al., 2017).

Results: For final cryo-EM map refinement, 140,000 particles were used resulting in $2.7 \AA$ resolution estimated using an $\mathrm{FSC}=0.143$ gold-standard threshold. The obtained structural data clearly demonstrate the peculiarities of the spatial organization of the $40 \mathrm{~S}$ ribosomal subunit, like the motility of the head relative to the body revealed by $3 \mathrm{D}$ variability analysis.

Conclusion: The resulting structure was solved at a significantly higher resolution compared to the previously published structure of a plant ribosome (Armache et al., 2010) and will be used as a reference for further studies of translation initiation in plants.

Key Words: cryo-EM・40S ribosomal subunit

This work was supported by the Russian Science Foundation (Grant No. 1974-20186)

*Corresponding author: Timur Baymukhametov.E-mail: baymukhametov.timur@gmail.com

International Journal of Biomedicine. 2021;11 Suppl 1: S20.

doi: 10.21103/IJBM.11.Suppl_1.P20

(C)2021 International Medical Research and Development Corporation 